\title{
A FENOMENOLOGIA E A PESQUISA DOS ESPAÇOS DE SERVIÇOS
}

\author{
José Luis Felicio Carvalho \\ E-mail: zkcarvalho@hotmail.com \\ Sylvia Constant Vergara \\ Professora Titular da FGV-EBAPE. \\ E-mail: vergara@fgv.br
}

Doutorando em Administração de Empresas pela PUC-RJ.

\begin{abstract}
RESUMO
A compreensão das experiências interativas e das vivências essenciais dos consumidores com os ambientes físicos de serviços não parece estar sendo adequadamente suprida por meio dos procedimentos metodológicos convencionais. No entanto, a recente emergência da perspectiva epistemológica da fenomenologia, e sua conseqüente metodologia em Marketing, vem constituindo-se como opção particularmente interessante e promissora para propiciar um melhor conhecimento daquelas situações. Embora seja assim, essa abordagem metodológica tem encontrado poucas formalizações acadêmicas capazes de ampliar seu uso por pesquisadores. Dessa forma, a proposta do presente ensaio é apresentar argumentos, procedimentos e formas pelas quais a fenomenologia pode constituir-se como opção metodológica adequada para a pesquisa dos espaços físicos de serviços.
\end{abstract}

ABSTRACT

Conventional methodological approaches fall short of being able to provide an adequate understanding of the prime interactive experiences customers undergo in service physical environments. Recently, the acceptance of phenomenology as an emergent approach to marketing issues opens both an interesting as well as fruitful alternative to investigate those encounter situations. Despite these promising conditions, practical guidelines

for a better use of phenomenology by marketing authors seem to be missing. This paper presents some procedures and indications that may help justifying phenomenology as a convenient methodological option to foster research on service physical environments.

\section{PALAVRAS-CHAVE}

Fenomenologia, pesquisa científica, espaços de serviços, interatividade, compreensão de significados.

\section{KEY WORDS}

Phenomenology, scientific research, servicescapes, interactivity, significant understanding. 


\section{INTRODUÇÃO}

A proposição de novos caminhos de pesquisa aplicada em Administração de Empresas implica uma mudança em relação à investigação científica que encontra no positivismo suas bases epistemológicas (Burrell e Morgan, 1979; Habermas, 1963; Pirsig, 1987; Vergara, 1989). Mudança esta que tem dominado tanto o campo de Marketing, privilegiado neste ensaio, quanto outras áreas da Administração (Cavedon, 2001; Ichikawa e Santos, 2001; Martins, 1996; Rossi e HorMeyll, 2001). A constatação de que grande parte da produção acadêmica no Brasil ampara-se em abordagens positivistas ou funcionalistas (Bertero et al., 1999) remete às recentes e preocupantes comparações das escolas brasileiras a fábricas de gestores capitaneadas pela "gerência científica" (Nicolini, 2001), aquela precipitadamente preocupada em prescrever ações universais, mais do que compreender o fenômeno observado e dessa compreensão extrair lições tanto teóricas quanto práticas.

A evidente predileção por parte dos acadêmicos brasileiros por enfoques de orientação positivista e de cunho eminentemente prescritivo, em detrimento de métodos de orientação fenomenológica, de cunho notadamente interpretativo, constitui uma dificuldade bastante significativa para a adoção da fenomenologia como prática efetiva de pesquisa. Nem os estudantes parecem estar adequadamente familiarizados com o desenvolvimento de pesquisas dessa natureza, nem os professores parecem sentir-se em posição confortável para participar mais ativamente daquelas investigações, seja como orientadores acadêmicos, seja como avaliadores do trabalho final de curso (Lima, 1999).

Quando se trata da área de Marketing, as dificuldades parecem potencializar-se (Brownlie e Saren, 1992; Révillion, 2001; Robson e Rowe, 1997; Zaltman, 2000). Veja-se, por exemplo, que em pesquisas que envolvem o consumidor, os métodos interpretativos, naturalmente raros, costumam basear-se nas tradições da etnografia, da semiótica aplicada ou do estruturalismo (Szmigin e Foxall, 2000). Apesar de muitas dentre essas abordagens incluírem por vezes uma perspectiva congruente à fenomenologia, elas não são, per se, metodologias fenomenológicas (Thompson et al., 1989).

A fenomenologia parece, por ora, condenada ao uso por apenas um pequeno grupo de pesquisadores. Entretanto, se ela parece ser capaz de reunir tantas vantagens, como se pretende aqui argumentar, talvez não seja interessante que os investigadores de Marketing conformem-se com sua aplicação irregular, esparsa, nem sempre criteriosa e restrita somente a alguns estudos esporádicos. Acredita-se, por outro lado, que a maior dificuldade encontrada na utilização dessa abordagem epistemológica consiste em que seus procedi- mentos metodológicos carecem de maior sintetização para uso de acadêmicos e estudantes ligados à área de Marketing.

Visando preencher a lacuna percebida, o presente ensaio - classificado como estudo metodológico (Vergara, 2000) - tem seu objetivo definido como o de fornecer indicações de procedimentos metodológicos básicos para os pesquisadores de Marketing de Serviços interessados em construir pesquisa acadêmica focada nos espaços físicos de serviços e fundamentada em perspectiva fenomenológica.

\section{A FENOMENOLOGIA PARECE CONDENADA AO USO POR}

\section{APENAS UM PEQUENO GRUPO DE "INICIADOS".}

O artigo está estruturado em oito seções, além desta Introdução. Em um primeiro momento, justifica-se a construção do presente ensaio. A seguir, detalha-se a demarcação científica da fenomenologia. Adiante, são exemplificados problemas de investigação e argumenta-se sobre a não-formulação de hipóteses. Na quinta seção, são apresentadas as diretrizes para amostragens e seleção de sujeitos. Na sexta, são fornecidas indicações para coleta de dados. A seguir, enfoca-se o tratamento dos dados de pesquisa. A oitava seção lista algumas limitações e dificuldades da metodologia. A última, à guisa de conclusão, reforça a motivação deste trabalho e a síntese dos pontos abordados.

\section{A JUSTIFICATIVA PARA O PRESENTE ENSAIO}

Conhecer o fenômeno de serviços, compreender sua natureza e entender as ferramentas que permitem seu aproveitamento constituem alguns dos atuais desafios para a ciência da Administração (Carvalho e Motta, 2000; Farias et al., 2000). A visão do marketing interativo - gestão das microssituações de encontros entre consumidores e prestadores de serviços como alicerce para a construção gradativa da satisfação do cliente fundamenta-se na premissa de que o valor maior criado pela empresa origina-se das experiências interativas propiciadas pelo fenômeno de serviços, durante a entrega da oferta da organização a seu público-alvo (Grönroos, 1995; Lovelock e Wright, 2001).

Por ser assim, o estudo dos momentos de relacionamento interativo entre os clientes e a empresa prestadora de serviços (Carvalho e Vergara, 2000; Motta, 2000; Solomon, 1998; Zeithaml e Bitner, 1996) vem 
ganhando o interesse crescente dos acadêmicos de Marketing. Esses momentos interativos, cruciais e decisivos para o sucesso mercadológico da organização de serviços, chamados de "horas da verdade" ou "momentos da oportunidade" (Normann, 1993), são os instantes em que se dá a experiência mais vívida do serviço. Essas horas são aquelas em que a organização tem uma oportunidade única de diferenciar sua oferta e constituem, portanto, um campo de trabalho científico especialmente interessante e promissor, não só pelo que pode agregar ao conhecimento científico, quanto pela sua utilidade prática.

Veja-se, por exemplo, a influência das condições físicas do ambiente de serviços nos processos de compra e consumo durante os "momentos da verdade" (Bitner, 1992; Zeithaml, 1988). Tal influência vem sendo reconhecida como fator importante para diferenciação mercadológica (Carvalho e Motta, 2000; Donovan e Rossiter, 1982; Motta, 2000; Oakes, 2000). O cenário oferecido pela empresa prestadora de serviços a seus públicos interno e externo - o ambiente físico da organização e os diversos elementos que compõem os espaços de serviços - proporciona o pano de fundo para a performance do serviço, da mesma forma que se faz para uma produção teatral (Grove et al., 1998; Lovelock e Wright, 2001).

No palco de serviços, o desempenho dos atores e o cenário são tão relevantes quanto nos espetáculos artísticos (Motta, 2000). Os espaços de serviços englobam o cenário e seus elementos tangíveis e intangíveis, incluindo, portanto, a atmosfera ou as condições ambientais que propiciam, a decoração, os objetos de cena, os efeitos especiais, a organização espacial, as qualidades de layout que oferecem e as significações que despertam (Grove et al., 1998).

A percepção do consumidor está atenta para as características do ambiente físico que afetam sua satisfação. Todos os aspectos ambientais capazes de serem detectados pelo aparelho sensório - tais como temperatura, luz, odor, barulho, música - podem interferir na interação dos clientes com a organização e seus funcionários (Carvalho e Motta, 2000; Creighton, 1998; Oakes, 2000). O estudo dos cenários de serviços comporta uma concentração de intenções, impressões, atitudes, propósitos e vivências que aparecem com base na experiência dos participantes do encontro de serviços (Sherry, 1998). Tal experiência implica a essência das significações atribuídas por esses indivíduos aos serviços e está longe de ser adequadamente explicada.

Mesmo tomando-se apenas a parte concreta, física ou tangível presente nas experiências de serviços, os efeitos reais desse ambiente continuam pouco explorados, às vezes inadequadamente conceitualizados e suas implicações nos comportamentos de compra carecem de aprofundamento científico. Os estudos dos espaços de serviços (ou cenários), que compreendem tanto as condições físicas do ambiente quanto o processo interativo entre clientes e empresa prestadora de serviços, precisam, enfim, de mais proposições para sua compreensão (Bitner, 1992; Carvalho e Motta, 2000; Donovan e Rossiter, 1982; Levy e Weitz, 2000).

Ainda que se considere a fase mais fértil da produção acadêmica internacional em serviços, a primeira metade da década de 1980, nos Estados Unidos e na Europa (Brown et al., 1994), durante a qual foi gerada a maior parte dos modelos hoje amplamente utilizados por todo o mundo, subsistem lacunas teóricas não preenchidas. Por mais que dessa fase tenham nascido contribuições importantíssimas para a compreensão do problema específico dos espaços de serviços, como é o caso do artigo seminal de Bitner (1992), talvez novos métodos de investigação científica possam desvendar facetas ainda não reveladas do fenômeno, principalmente quando se trata do desenvolvimento de novos conhecimentos (Sherry, 1998), e não somente da testagem do saber acadêmico que atualmente já se encontra sedimentado.

Argumenta-se, portanto, aqui, que as pesquisas em ambientes de serviços alinhadas com os métodos científicos dominantes, embora relevantes, não têm conseguido gerar pesquisas acadêmicas capazes de abarcar a gestalt do fenômeno. O processo de mensuração comumente usado é, freqüentemente, incompleto. Os enfoques de estudo são demasiadamente estreitos e a testagem de hipóteses, bastante utilizada, torna-se complicada ou confusa (Goulding, 1999; Prus, 1991; Rossi e Hor-Meyll, 2001; Sherry, 1998).

O estudo de fenômenos complexos, como aqueles que ocorrem nas "horas da verdade", demanda um tratamento multidimensional, transdisciplinar e interrelacional (Carvalho e Vergara, 2000; Malhotra e Peterson, 2001; Serva e Jaime Jr, 2001; Weil et al., 1993), como o que propicia o método fenomenológico. Sugere-se, então, que a demarcação científica a ser seguida para a formalização do trabalho de pesquisa nos ambientes de serviços, para a coleta e para a interpretação dos dados, procure fugir à tradição realista $\mathrm{e}$ objetivista do método hipotético-dedutivo, de base positivista (Burrell e Morgan, 1979).

Seguindo a concepção acadêmica que anuncia uma nova era para o conhecimento da ciência do Marketing (Brown, 1997; Brownlie, 1997; Brownlie et al., 1999; Casotti, 1999), alguns acadêmicos dessa área vêm-se propondo a enfatizar pesquisas alinhadas com o método fenomenológico-hermenêutico (Creswell, 1998; Foxall, 1995; Goulding, 1999; Jackson, 1996; Kerlinger e Lee, 2000; Morse, 1994; Patton, 1990; Ray, 1994; Remenyi et al., 1998) e amparadas pela perspectiva epistemológica presente no conjunto de teorias identificadas com a ideologia pós-moderna (Alvesson e 
Deetz, 1999; Brown, 1997; Chia, 1995; Kilduff e Mehra, 1997).

A inserção, na investigação fenomenológica, do interacionismo simbólico, dos estudos antropológicos e socioculturais, das teorias dos psicólogos ambientais, dos trabalhos de arquitetos, designers e decoradores, assim como da perspectiva pós-moderna, caracteriza a emergência recente de uma nova abordagem em pesquisa de Marketing (Sherry, 1995; 1998): a "fenomenologia dos ambientes mercadológicos". Ela é definida da maneira como segue (Sherry, 1995, p. 439, tradução livre):“Em um primeiro nível, esta tradição está preocupada com os comportamentos humanos nos locais das experiências, e examina questões que nascem diretamente das instituições formais e informais. Em um outro nível, mais abstrato, a perspectiva está voltada para os mundos caleidoscópicos individuais que são habitados pelos stakeholders da empresa durante as transações de marketing. O primeiro aspecto lida com o impacto dos espaços físicos no comportamento adotado nos cenários de mercado; o segundo lida com a elaboração individual do ambiente como um campo para a projeção de fantasias pessoais. Cada aspecto desses contribui para o estado de experiência que identificamos como "estar no local de marketing"'.

Os ambientes mercadológicos adequam-se a problemas de estudos que tenham como fulcro a compreensão das múltiplas e complexas dimensões das experiências interativas vividas pelas pessoas envolvidas com as evidências físicas ou com os espaços de serviços. Exemplos são os problemas de investigação que focalizam o exame da percepção de consumidores em relação aos estímulos ambientais em shopping centers (Sandkici e Holt, 1998), ou a investigação dos aspectos criativos do consumo possibilitados por layouts de lojas de varejo (Creighton, 1998).

\section{DEMARCAÇÃO CIENTÍFICA}

A abordagem fenomenológica consiste em um método subjetivista de investigação científica (Burrell e Morgan, 1979). Pressupostos e julgamentos são abandonados, permitindo que o conhecimento possa nascer da experiência do pesquisador com a essência de seu objeto de pesquisa (Creswell, 1998; Masini, 1989; Ray, 1994). Na visão de Husserl (1970), é próprio do método o abandono, pelo pesquisador, de idéias preconcebidas. Ele rejeita a abordagem positivista, a concretude, a preocupação nomotética, a ênfase quantitativa e a imagem do cientista isolado e puramente observador (Gil, 1987; Kerlinger e Lee, 2000; Vergara, 2000).

As três tradições fenomenológicas predominantes são: (a) a escola transcendental ou descritiva, criada por Husserl (1970); (b) a abordagem interpretativa ou hermenêutica, desenvolvida por Heidegger (1962), aluno e crítico de Husserl; e (c) a perspectiva de integração, que busca reunir as duas primeiras tradições, e que ganhou força a partir dos anos 1990 (Masini, 1989; Ray, 1994; Van Manen, 1990). A fenomenologia dos ambientes de serviços busca um alinhamento com essa terceira possibilidade integrativa, ainda que pretenda reservar um peso maior à hermenêutica do que à transcendência.

\section{A fenomenologia mostra-se}

\section{PARTICULARMENTE INTERESSANTE}

\section{PARA EXAMINAR AS PERCEPÇÕES}

\section{DOS PARTICIPANTES DE UMA}

\section{INTERAÇÃO DE SERVIÇOS.}

Quando o método fenomenológico pratica a hermenêutica, busca entender o fenômeno observado, interpretá-lo, perceber seu significado (Vergara, 2000). Assim, pode-se falar em um método fenomenológicohermenêutico, estudo científico das experiências humanas (Jackson, 1996; Remenyi et al., 1998; Van Manen, 1990) que descreve os significados das vivências experimentadas por determinados indivíduos em relação a um dado fenômeno (Creswell, 1998). A abordagem fenomenológica não se preocupa em estudar, portanto, objetos e atores sociais isolados; a tarefa do pesquisador é buscar compreender como a realidade se constrói por meio da experiência das pessoas envolvidas em determinada situação ou com um dado fenômeno. Como diria Heidegger (apud Morente, 1980, p. 310), é o "estar eu com as coisas do mundo".

Em virtude de seu foco na consciência humana durante a vivência de situações sociais e de ser, provavelmente, a metodologia que melhor consegue capturar a essência da experiência interativa humana com os ambientes (Baker, 1998), a fenomenologia parece, portanto, particularmente interessante para examinar as percepções dos participantes de uma interação de serviços (Goulding, 1999; Sherry, 1998; Thompson et al., 1989). Suas teorias são derivadas de maneira indutiva, quando são agregadas as padronizações permitidas pela interpretação dos significados da experiência (Churchill e Wertz, 1985; Foxall, 1995; Gil, 1987; Ichikawa e Santos 2001).

O estudo dos ambientes de serviços não se ampara, porém, exclusivamente na tradição fenomenológica pura; ao contrário, pede-se que a perspectiva pós-moderna seja agregada à sua metodologia. Embora seja muito difícil definir o conceito de pós-moderno (Allan, 
2000; Creswell, 1998; Sokal e Bricmont, 1999), sabese que essa abordagem emergiu para o interesse acadêmico na área organizacional por volta dos anos 1980 (Alvesson e Deetz, 1999). Tanto a fenomenologia quanto a perspectiva pós-moderna têm seu foco central na essência do fenômeno analisado (Chia, 1995).

Pelas vantagens oferecidas, a abordagem pós-moderna do consumo vem sendo utilizada para o estudo teórico e para a compreensão prática dos ambientes físicos de marketing (Aubert-Gamet e Cova, 1999; Gottdiener, 1994, 1995, 1998; Kilduff e Mehra, 1997). No que concerne à pesquisa em comportamento do consumidor, redireciona seu foco para as situações ou rituais - de consumo e suas vizinhanças físicas, preocupando-se com a análise do processo de compra, complexo e virtualmente imprevisível (Casotti, 1999; Cova, 1996, 1997).

\section{DO PROBLEMA DE INVESTIGACC̃̃O À NÃO-FORMULAÇÃO DE HIPÓTESES}

Após a definição do problema de pesquisa, padrões convencionais de investigação empírica, geralmente, definem hipóteses - entendidas como respostas antecipadas ao problema. Em geral elas exigem testes de formulação estatística (Botelho e Macera, 2001). No entanto, essa forma de se chegar à solução de um problema não é a única possível e não se aplica a todas as áreas ou situações, sobretudo quando está em jogo o contexto impreciso da pesquisa social. A elaboração de testes de hipótese pode ser vista como uma técnica estatística de aplicação restritiva, dificultadora de uma compreensão integrada do problema - que, às vezes, pode funcionar como entrave à criatividade do pesquisador e, por esse mesmo motivo, como dificultador do exercício do próprio espírito científico: a descoberta.

Uma vez que se rejeite a metodologia hipotéticodedutiva, amparada na epistemologia positivista, não faz sentido a formulação e a experimentação de hipóteses (Allan, 2000; Remenyi et al., 1998).

Enquanto a pesquisa acadêmica alinhada com uma visão cartesiana ou positivista da ciência tem como enfoque a redução de um fenômeno a dimensões quantitativas que aderem a leis e princípios, a perspectiva fenomenológica demanda de seus pesquisadores uma descrição interpretativa do fenômeno (Casotti, 1999). Assim, sua lógica não busca a experimentação de padrões previamente estruturados, mas, ao contrário, tem como base racional a construção de uma dinâmica de pesquisa focada na experiência interativa humana contada na primeira pessoa (Cavedon, 2001), para que se possa visualizar padrões extraídos do mundo dos sujeitos da pesquisa à medida que eles emergem (Churchill e Wertz, 1985; Helgeson, 1994; Malhotra e
Peterson, 2001; Mendonça, 2001; Patton, 1990; Prus, 1991; Thompson et al., 1989).

O método fenomenológico não estimula os pesquisadores a encontrar uma verdade definitiva para seus problemas de estudo. A fenomenologia é, antes de tudo, uma abordagem inacabada; o fenômeno investigado sempre poderá ser retomado e visto sob nova interpretação. Diferentemente do que às vezes faz crer o método hipotético-dedutivo, o fenomenológico pressupõe um recomeçar incessante, um enfoque que recusa cristalizações em sistemas acabados e fechados (Masini, 1989).

A abordagem pós-moderna, por seu turno, assume que o consumidor manifesta uma nítida desordem em seus padrões de compra, sendo impossível tentar prever seu comportamento dentro da visão cartesiana (Brown, 1993; Cova, 1996; 1997; Mills, 1982; Philips, 1995). Para analisar o processo de consumo, a pesquisa concentra-se nos rituais de compra e em seus cenários físicos, abandonando as tentativas de classificar os comportamentos em padronizações preestabelecidas (Cova, 1997; Cova e Badot, 1995). A lógica formal clássica seria de pouca valia para lidar com conhecimentos cujas características são principalmente informais e obtidas em situação de comunicação interativa (Helgeson, 1994). Sendo assim, o pesquisador dos ambientes mercadológicos não precisa temer a questão da imprecisão, nem deve acreditar que processar qualitativamente a informação obtida em situação de interação comunicacional constitui uma infração contra a ciência (Cavedon, 2001; Ichikawa e Santos, 2001; Lima, 1999).

Por outro lado, ainda que a metodologia fenomenológica não pretenda antecipar respostas prévias aos problemas de estudo (Ichikawa e Santos, 2001; Mills, 1982; Ray, 1994), espera-se que a análise, a interpretação e a discussão dos resultados eventualmente obtidos em trabalhos de campo permitam que sejam sugeridas algumas proposições para guiar o desenvolvimento de hipóteses testáveis em futuros trabalhos acadêmicos que optem pelo método hipotético-dedutivo (Allan, 2000; Creswell, 1998), uma vez que acessar o conhecimento por determinado método não significa que aquele conhecimento não poderá ser aproveitado por adeptos de outros métodos. Estabelece-se, assim, a possibilidade de complementaridade de métodos.

Apesar da rejeição, pelos adeptos da fenomenologia, da lógica exclusivamente hipotético-dedutiva, os autores do presente ensaio reconhecem, por outro lado, que existem evidências abundantes de que a melhor maneira de acelerar-se o desenvolvimento científico implica o uso tanto de indução quanto de dedução (Reynolds, 1971). Essa complementaridade traduzirse-ia tanto na indução criativa realizada pela fenomenologia, em que o pesquisador parte dos fatos para a 
teoria, quanto pela dedução implementada no teste de hipóteses, que parte da teoria para os fatos. Os dois movimentos - dedução e indução - poderiam ser vistos, antes de tudo, como duas faces essenciais do esforço científico, inerentes às lógicas de descoberta e justificação (Bunge, 1998).

\section{UNIVERSO, AMOSTRA E SELEÇÃO DOS SUJEITOS}

Os cenários de serviços ainda são largamente orientados para a maximização funcional do espaço e para a satisfação de um consumidor isolado. No entanto, de acordo com a perspectiva pós-moderna, se os cenários de serviços assumirem um papel de suporte social, eles poderão ser percebidos como os lugares favoritos dos grupos subculturais urbanos para a consagração de seus rituais de integração e reconhecimento (Cova, 1996, 1997; Gottdiener, 1994, 1995; Schouten e McAlexander, 1995). O caso dos shopping centers é emblemático.

$\mathrm{Na}$ sociedade pós-moderna, o comportamento de consumo delineia-se, sobretudo, como um processo de aquisição de símbolos e imagens percebidas em um produto ou serviço, por meio da posse do qual o indivíduo identifica-se perante um grupo de referência. A expressão "tribo urbana" traduz a caracterização mais apropriada para os segmentos de mercado que a fenomenologia dos ambientes mercadológicos pretende estudar: comunidades de consumidores nas quais a união de seus membros dá-se por laços emocionais, estilos de vida, identificação com subculturas e práticas de consumo comuns (Chia, 1995; Schouten e McAlexander, 1995).

Os ambientes de serviços aos quais acorrem os clientes representantes do tribalismo urbano, geralmente, comportam cenários temáticos (Creighton, 1998; Gottdiener, 1998; Sandkici e Holt, 1998), que funcionam como palco para o espetáculo da comunicação de consumo tribal.

Tribos urbanas apontam para outra preocupação comum aos pesquisadores pós-modernos e estudiosos que adotam a metodologia fenomenológica. Para os adeptos da fenomenologia, a natureza dos participantes do estudo é de importância crucial - preferencialmente, essa abordagem indica, como sujeitos de pesquisa, indivíduos ou grupos de características singulares e que estejam envolvidos em experiências similares (Patton, 1990; Ray, 1994; Van Manen, 1990).

Considerando-se o universo das tribos de consumidores, as populações amostrais da fenomenologia dos ambientes mercadológicos são selecionadas, de acordo com critérios de representatividade não probabilísticos, por tipicidade (Creswell, 1998; Vergara, 2000) e por conveniência (Motta, 1999). A exigência da tipicidade é satisfeita pela seleção de consumidores considerados representativos das tribos urbanas características dos ambientes específicos que se pretende investigar. Já a conveniência é responsável pela seleção das pessoas que se encontram no ambiente de serviços no momento da observação de sua interação com o cenário da empresa (Arnould e Price, 1993; Motta, 1999), uma vez que as medidas obtidas no instante da ocorrência do fenômeno interativo (Gil, 1987) servem para uma avaliação mais imediata e espontânea dos sentimentos do consumidor.

\section{Pesquisadores PÓS-MOdeRnos DEFENDEM QUE O MARKETING} PROCURE INFLUENCIAR O CONSUMIDOR COM EXPERIÊNCIAS E VIVÊNCIAS CUJA ESSÊNCIA SEJA A PARTICIPAÇÃO.

Quanto às amostras adequadas para a fenomenologia dos ambientes mercadológicos, a metodologia advoga que não existem regras específicas capazes de estabelecer com precisão o tamanho de uma população amostral (Kerlinger e Lee, 2000; Motta, 1999). Foram registrados estudos qualitativos de base fenomenológica com números de entrevistados que variavam de uma a 325 pessoas (Creswell, 1998).

\section{COLETA DE DADOS}

A relação do método fenomenológico com a teoria constrói-se em dois momentos principais (Churchill e Wertz, 1985; Morse, 1994; Ray, 1994): (a) quando as teorias guiam o processo de pesquisa, principalmente nas fases iniciais de estudo, ou seja, quando se procura montar um quadro teórico de referência; e (b) quando, finalmente, as teorias emergem do processo reflexivo propiciado pela análise dos dados do trabalho.

Além de manter uma preocupação permanente em compreender a essência de uma dada situação social, a pesquisa fenomenológica também deve procurar preencher as lacunas presentes na teoria existente, tarefa que costuma exigir extensas revisões de literatura (Creswell, 1998; Miles e Huberman, 1994; Patton, 1990).

Com relação aos estudos de campo, a metodologia fundamenta suas discussões na obtenção de medidas perceptuais subjetivas (Kerlinger e Lee, 2000; Motta, 
1999; Révillion, 2001) com os participantes dos encontros de serviços. O pressuposto aqui defendido baseiase nas idéias de que: (a) o aprendizado científico "envolve um processo gradual de entendimento dos significados e das experiências vividas pelos pesquisados" (Santos, 1994, p. 33); e (b) os consumidores são os melhores avaliadores de suas experiências individuais, "uma vez que suas percepções constituem uma realidade própria, cujo alcance é limitado para avaliadores externos" (Motta, 1999, p. 3).

A metodologia aqui proposta defende uma abordagem rigorosa da pesquisa qualitativa, com a utilização sistemática de procedimentos científicos, em uma dinâmica que envolve a obtenção e a interpretação de material empírico, coletado e analisado por meios diversos (Casotti, 1999; Sherry, 1998; Thorne, 2001). Abole-se o fetichismo do método e da técnica, de qualquer norma de procedimento cuja rigidez cause entraves à reflexão e à imaginação do pesquisador (Lima, 1999; Milliken, 2001; Mills, 1982).

Geralmente, a abordagem fenomenológica abraça uma sequiência básica de passos para a coleta e para o tratamento dos dados (Creswell, 1998; Moustakas, 1994), conforme pode ser visualizado na Figura 1.
É fácil perceber que os meios de coleta de dados, sob o enfoque fenomenológico, costumam comportar, basicamente, a observação participante e as entrevistas semi-estruturadas nos cenários das situações sociais do mundo real (Kerlinger e Lee, 2000; Santos, 1994; Sherry, 1998). Em outras palavras, os objetivos da pesquisa orientam as questões propostas, mas seu conteúdo, sua seqüência e sua enunciação estão nas mãos do entrevistador, no momento mesmo em que investiga. Quando se elege como metodologia de pesquisa a abordagem fenomenológica, questionários fechados são inapropriados (Vergara, 2000). Nas entrevistas, as perguntas fluem, em acordo com a idéia de que "a arte de perguntar é a arte de pensar" (Ray, 1994, p. 129, tradução livre). Muita instrumentalização prévia costuma fechar os olhos do estudioso para a essência do fenômeno a ser examinado (Miles e Huberman, 1994; Rossi e Hor-Meyll, 2001). O pesquisador deve dirigir sua atenção para as transações sociais e para as interações entre pessoas e eventos, sob uma ótica naturalista, participativa e interpretativa (Ichikawa e Santos, 2001; Kerlinger e Lee, 2000; Mendonça, 2001; Prus, 1991; Révillion, 2001).

A entrevista é uma situação de interação face a face em que uma pessoa - o entrevistador - propõe a outro

Figura 1 - Passos para a coleta e o tratamento dos dados

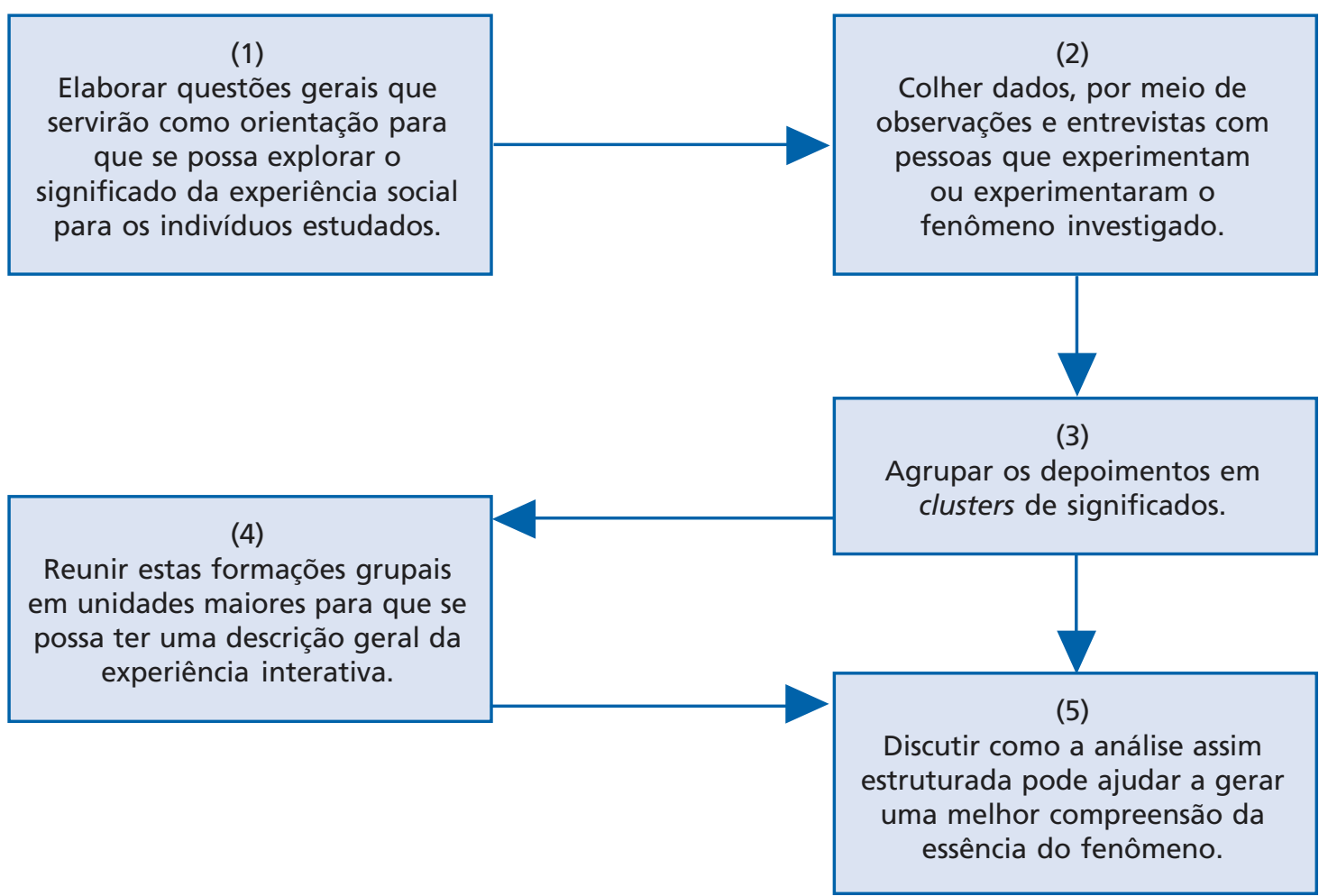


indivíduo - o entrevistado - questões destinadas a obter respostas pertinentes ao problema de pesquisa (Gil, 1987; Kerlinger e Lee, 2000; Vergara, 2000). A perspectiva aqui pretendida entende a entrevista como uma interação em que existe uma relação ativa de tomada de papéis, na qual o entrevistador comporta-se como um instrutor que conduz o rumo da conversa, mas deixa espaço para o desenvolvimento do papel do entrevistado (Kerlinger e Lee, 2000).

Entrevistas não padronizadas, semi-estruturadas não são, de maneira alguma, casuais. Elas devem ser tão cuidadosamente planejadas quanto os questionários estruturados (Thorne, 2001). A meta de uma entrevista fenomenológica é obter uma descrição, em primeira pessoa, de uma experiência, a partir da qual serão detectados padrões experimentais capazes de gerar uma compreensão essencial do fenômeno. A entrevista, no entanto, deve manter a estrutura de uma conversa, não de uma sucessão linear de perguntas e respostas. $\mathrm{O}$ curso do diálogo depende do entrevistado e do entrevistador. O diálogo tende a ser circular, ou seja, o entrevistador permite que o entrevistado construa livremente suas respostas, mas procura reconduzir o foco da conversa para o tema da pesquisa (Gil, 1987; Thompson et al., 1989).

A condução das entrevistas demanda do entrevistador uma postura de observador participante (Arnould e Price, 1993; Kerlinger e Lee, 2000), uma vez que se busca interferir no comportamento das pessoas no ambiente, chamando sua atenção para determinado estímulo ambiental, por exemplo, ou pedindo que o indivíduo modifique o rumo de sua narrativa. Propõe-se, mais especificamente, que o pesquisador adote uma atitude de espectador interativo (Vergara, 2000), capaz de integrar-se ao grupo pesquisado, porém sem buscar desempenhar o papel de um membro pertencente àquela comunidade.

Por meio dessa dinâmica, a pesquisa obtém depoimentos, histórias vivas e narrativas (Arnould e Price, 1993; Brownlie, 1997; Philips, 1995), em lugar das hipóteses e das estatísticas características da tradição positivista (Kilduff e Mehra, 1997). A interação dos consumidores com os ambientes físicos das organizações materializa-se em relatos contados sob a ótica dos participantes da experiência, não dos que a idealizaram.

O método fenomenológico demanda que a experiência manifestada como linguagem pelos participantes do estudo seja vista como principal insumo do processo da pesquisa (Van Manen, 1990; Ray, 1994); a palavra dos sujeitos pesquisados deve ser fonte primária de dados (Morse, 1994; Santos, 1994). Tais dados devem ser capturados, transcritos como texto e, então, estarão sujeitos à abordagem escolhida pelo pesquisador - descrição, interpretação, ou ambas - e que irá determinar a natureza da análise (Ray, 1994), como se passa a expor na próxima seção.

Cabe observar que a fase de coleta de dados - denominada "etapa de compreensão" pelos seguidores da fenomenologia - pode ser considerada como cumprida quando o pesquisador colecionou informação suficiente

\section{A fENOMENOLOGIA É, ANTES}

\section{DE TUDO, UMA ABORDAGEM \\ INACABADA; O FENÔMENO \\ INVESTIGADO SEMPRE PODERÁ}

\section{SER RETOMADO E VISTO SOB}

\section{NOVA INTERPRETAÇÃO.}

para estar apto a redigir uma descrição detalhada, rica e coerente da experiência observada. O compreender, passo inicial para a dinâmica de aprendizagem fenomenológica dos ambientes mercadológicos, significa, então, reunir exaustivamente dados acerca de uma experiência, principalmente sobre seus participantes e sobre o ambiente físico em que o fenômeno ocorre (Morse, 1994).

\section{TRATAMENTO DOS DADOS}

Como mencionado, os dados coletados não podem ser tratados por procedimentos estatísticos e remetem à dinâmica qualitativa de pesquisa, em que tanto as perguntas quanto as respostas rejeitam padrões (Miles e Huberman, 1994; Milliken, 2001).

Estudos qualitativos são processos de investigação elaborados para possibilitar uma melhor compreensão de uma dada problemática social em contextos locais (Creswell, 1998; Remenyi et al., 1998; Santos, 1994). O pesquisador esboça um retrato complexo e holístico da situação, analisando narrativas e depoimentos dos atores sociais, conduzindo seu estudo em um ambiente real.

A pesquisa qualitativa emana, então, da própria escolha por uma perspectiva fenomenológica (Churchill e Wertz, 1985; Milliken, 2001; Patton, 1990; Ray, 1994; Santos, 1994; Sherry, 1998) e caracteriza-se por três fundamentos principais: (a) o conhecimento é construído ativamente e surge do exame dos mecanismos perceptuais individuais, manifestados na linguagem; (b) o pesquisador confia em uma dinâmica de observação e busca manter intactas as perspectivas dos participantes; e (c) o investigador procura descrever e interpretar - principalmen- 
te pela via da semiótica - as formas pelas quais as pessoas relacionam experiências, linguagem, comportamentos e seus significados (Gottdiener, 1994; Kilduff e Mehra, 1997; Rossi e Hor-Meyll, 2001).

Vale sempre ressaltar que a base metodológica da fenomenologia dos ambientes mercadológicos tem sua proposta epistemológica voltada para: (a) adentrar o campo da experiência humana; (b) focar a percepção da situação social pelos participantes; (c) ver como o fenômeno investigado desdobra-se a partir da experiência social; (d) observar o significado das experiências dos participantes; e (e) entender a vida como um mistério e não como um problema a ser resolvido (Creswell, 1998).

\section{MUITO PODE SER GANHO}

\section{QUANDO SE PERMITE AOS}

\section{CONSUMIDORES, GERENTES,}

\section{VENDEDORES E DEMAIS}

\section{PARTICIPANTES DO ENCONTRO}

\section{DE SERVIÇOS "DIZER ALGO}

\section{QUE NÃO SEJA IMEDIATAMENTE DOMESTICADO POR TEORIAS".}

A abordagem hermenêutica considera duas questões como essenciais para que sejam compreendidas as formas pelas quais as pessoas interagem: a linguagem e a semiótica (Ichikawa e Santos, 2001; Mendonça, 2001; Ray, 1994). Os fenômenos nascidos da experiência humana só podem ser interpretados por intermédio da maneira pela qual os indivíduos constróem suas narrativas. O entendimento é mediado, então, pela interpretação dessa linguagem. Existe, portanto, uma relação muito íntima e articulada entre fenomenologia, hermenêutica e semiótica; não se pode acessar a essência do fenômeno sem endereçar, via interpretação, a experiência humana e seu significado (Van Manen, 1990). A fenomenologia suspende, portanto, o exame dos determinantes subjacentes às crenças dessas pessoas, para procurar descrever as implicações, as intenções e os efeitos daquilo que elas dizem, fazem e acreditam que seja verdadeiro (Creswell, 1998; Goulding, 1999; Sherry, 1995, 1998).

Em relação à orientação epistemológica, a abordagem de pesquisa aqui privilegiada evoca a visão de que muito pode ser ganho quando se permite aos consumidores, gerentes, vendedores e demais participantes do encontro de serviços "dizer algo que não seja imediatamente domesticado por teorias" (Alvesson e
Deetz, 1999, p. 259). Mobiliza-se igualmente a preocupação em deixar que os consumidores ou os gerentes falem por si mesmos, ainda que seja difícil para o pesquisador compreender o que está sendo dito (Brownlie et al., 1999). Defende-se também o entendimento de que os acadêmicos ganham em comportarse mais como intérpretes do que como legisladores (Brown, 1997; Szmigin e Foxall, 2000), assim como se defende o imperativo de produzir novas formas de conhecimento que só podem ser obtidas quando são transpostas as rígidas fronteiras disciplinares (Kilduff e Mehra, 1997; Weil et al., 1993).

Finalmente, propõe-se uma postura de humildade face às evidências empíricas, especialmente em relação aos eventuais efeitos anômalos que poderiam contradizer crenças estabelecidas. Defende-se a postura do pesquisador como um "artesão intelectual", que se entrega carinhosamente às suas experiências, desenvolve cuidadosamente suas reflexões e depois as põe à prova, deixando sempre que sua mente transforme-se "num prisma móvel, colhendo luz de tantos ângulos quanto possível" (Mills, 1982, p. 231).

Mantendo em mente essas considerações epistemológicas, o pesquisador deve tratar os dados coletados. Depois que as entrevistas forem transcritas, se tiverem sido feitas gravações, a fase de interpretação tem início. Com os dados empíricos a seu dispor, o pesquisador fenomenológico deve praticar uma imersão no mundo descrito pelos entrevistados, buscando observar o fenômeno da forma como ele foi experimentado, procurando uma empatia com os participantes da experiência. Segue-se uma análise interpretativa profunda das entrevistas, que deve contar amplamente com a teoria que deu suporte à fase inicial do estudo (Churchill e Wertz, 1985; Helgeson, 1994), embora não seja sua escrava.

A análise dos dados qualitativos da fenomenologia dos ambientes mercadológicos costuma seguir, mais especificamente, dois procedimentos principais: (a) a leitura crítica; e (b) a classificação de termos e idéias (Motta, 1999). Uma vez que se pretende trabalhar com relatos e narrativas, é imprescindível que se faça inicialmente uma leitura crítica das transcrições das entrevistas, identificando os pontos que merecem atenção e articulando-os com as informações obtidas na revisão da bibliografia pertinente ao problema sob investigação. A análise é alimentada pela reflexão hermenêutica, que consiste na interpretação dos dados e textos de pesquisa como um movimento dinâmico em direção a uma melhor compreensão do fenômeno investigado (Casotti, 1999; Ray, 1994; Remenyi et al., 1998).

Para que se possa chegar à dinâmica geral do fenômeno observado com base no conjunto de depoimentos pessoais, as análises individuais são agrupadas de 
acordo com três possibilidades não-excludentes de formação de clusters: (a) padrões que seguem os termos usados pelos próprios depoentes; (b) padrões que emergem de coincidências observadas no texto; e (c) padrões previamente estipulados (Moustakas, 1994; Thompson et al., 1989). O objetivo do pesquisador, ao derivar categorias de clusters, deve ser tão somente o de apresentar o material aos leitores de modo que se mantenha, o máximo possível, a essência das experiências individuais (Prus, 1991).

Esse procedimento, a que os pesquisadores alinhados com a metodologia fenomenológica chamam "síntese", consiste na fusão de vários depoimentos, casos, histórias e relatos para descrever um padrão típico de comportamento ou de resposta (Morse, 1994), caracterizando uma construção "essencial" ou "universal" (Helgeson, 1994; Ray, 1994). Assim, por meio das análises temáticas (Morse, 1994), propiciadas pela categorização ou síntese dos depoimentos em clusters, identificam-se estruturas comuns à experiência particular, capazes de otimizar a interpretação dos dados de pesquisa.

\section{LIMITAÇÕES E DIFICULDADES NO USO DO MÉTODO}

$\mathrm{O}$ tratamento dos problemas de pesquisa nos ambientes mercadológicos pelo método fenomenológicohermenêutico traz as complicações decorrentes do fato de se estar usando, à guisa de objetos de pesquisa, seres humanos e suas percepções, seus sentimentos, suas impressões, seus significados. A observação científica da matéria-prima humana exige que se pague o preço da subjetividade e da incerteza (Creswell, 1998; Goulding, 1999; Miles e Huberman, 1994). Além disso, os métodos para tratamento e análise de dados qualitativos ainda não se encontram plenamente desenvolvidos (Miles e Huberman, 1994; Santos, 1994).

Se a capacidade de adentrar o mundo dos sujeitos pesquisados para conhecer os fenômenos por meio de seu olhar é uma das principais vantagens da fenomenologia, daí decorrem também duas graves limitações. A primeira diz respeito à habilidade do pesquisador em praticar esse olhar empático (Mendonça, 2001); a segunda relaciona-se à restrição das possibilidades de generalização dos resultados da pesquisa, em virtude da natureza peculiar das populações amostrais e das experiências singulares narradas aos pesquisadores (Botelho e Macera, 2001; Churchill e Wertz, 1985; Helgeson, 1994).

A inclusão de informações subjetivas no trabalho científico caracteriza um enorme desafio (Malhotra e Peterson, 2001; Szmigin e Foxall, 2000). A multiplicidade de experiências diversas percebida pela abordagem fenomenológica em um único ambiente de serviços não é somente recorrente, como também inevitável (Ray, 1994; Sherry, 1998). A principal dificuldade da fenomenologia, entretanto, está no fato de sua metodologia consistir em uma perspectiva geral de observação e de pensamento, ao invés de configurar uma teoria plenamente desenvolvida (Goulding, 1999; Masini, 1989). A fenomenologia pode ser considerada como uma filosofia, uma epistemologia, um método, ou os três simultaneamente (Ray, 1994).

\section{Defende-se A POSTURA}

\section{DO PESQUISADOR COMO UM}

\section{"ARTESÃo InTElectual", QUe} SE ENTREGA CARINHOSAMENTE ÀS

SUAS EXPERIÊNCIAS, DESENVOLVE CUIDADOSAMENTE SUAS REFLEXÕES

\author{
E DEPOIS AS PÕE À PROVA.
}

Outro entrave na pesquisa fenomenológica advém do fato de que lidar com dados qualitativos pede o desenvolvimento de "sensibilidade teórica", entendida como "a sensibilidade que se exige do pesquisador para reconhecer dados importantes e dar-lhes sentido" (Santos, 1994 , p. 105). Essa habilidade nasce da experiência profissional, da vivência pessoal e de conhecimentos adquiridos por meio de literatura especializada. Impõe-se, então, uma responsabilidade ingrata ao cientista ao se demandar que ele seja capaz de praticar uma autocrítica objetiva no sentido de avaliar se sua própria sensibilidade teórica já foi suficientemente desenvolvida para o trato adequado com a matéria de seu estudo.

O trabalho de observação participante com tribos de consumidores também pode trazer os riscos da nãoaceitação do pesquisador pelo grupo, da dificuldade de aproximação com os indivíduos, de conversas pouco produtivas em termos de informações relevantes ou mesmo da recusa de interação (Santos, 1994). A análise de depoimentos e narrativas dos participantes, por sua vez, é um método que incorpora igualmente as percepções e impressões do pesquisador, elementos que costumam comprometer a reprodução da integridade do discurso dos sujeitos da pesquisa (Brownlie, 1997; Kerlinger e Lee, 2000). Mas não há como evitar-se a subjetividade humana, sua própria essência (Mendonça, 2001).

A visão da pós-modernidade, por sua vez, vem re- 
cebendo críticas contundentes por parte dos partidários da idéia de que o campo humano e o social não podem ser apreendidos pela racionalidade científica, tal como a vêem, por exemplo, Sokal e Bricmont (1999). Dado que a fenomenologia dos ambientes mercadológicos está interessada basicamente no pensamento interpretativo da pesquisa pós-moderna para que possa explorar melhor os significados inerentes à atividade social e os padrões de interação social que caracterizam a experiência humana (Cova e Badot, 1995; Philips, 1995), foge àquela racionalidade. A ênfase está no desenvolvimento de uma compreensão do universo simbólico presente na ação organizacional (Allan, 2000; Prus, 1991), não em fórmulas e diagramas matemáticos ou na discussão de "comportamentos de pessoas que vão às compras da mesma forma que um cientista observa formigas em uma fazendinha de formigas" (Philips, 1995, p. 626).

O tema da abordagem fenomenológica, como se pode depreender das indicações metodológicas aqui sugeridas, possui diversas vertentes de discussão que mereceriam, certamente, um exame mais detido. De fato, não se espera, no âmbito de um só artigo, esgotar as muitas facetas de um assunto tão complexo. Para buscar suprir as eventuais lacunas deixadas pela escassez de espaço para aprofundamento da matéria, indica-se, por meio do Quadro 1, um leque de referências bibliográficas fundamentais que serviriam como alicerces complementares imprescindíveis ao leitor interessado em conduzir aplicações da fenomenologia em marketing de serviços. Muitas das indicações citadas aparecem igualmente nas Referências Bibliográficas, de onde também poderão surgir outras possibilidades de pesquisa.

\section{PARA CONCLUIR}

$\mathrm{Na}$ área da Administração em geral, e do Marketing em particular, o enfoque funcionalista e quantitativo, de orientação positivista, impõe sua supremacia, praticamente colocando-se como única possibilidade racional de pesquisa. Essa pretensa exclusividade científica legitima a exclusão de abordagens não-quantitativas e tende a tolher esforços mais ousados do pesquisador no sentido de avançar com menos timidez nos comple-

\section{Quadro 1 - Uma listagem fundamental de referências bibliográficas complementares}

BHASKAR, Roy. The possibility of naturalism: a philosophical critique of the contemporary human sciences. $2^{\text {nd }}$ ed. Hemel Hampstead : Harvester Wheatcheaf Press, 1989.

CHALMERS, Alan F. What is this thing called science? : an assessment of the nature and status of science and its methods. $3^{\text {rd }}$ ed. Milton Keynes : Open University, 1999.

CRESWELL, John W. Qualitative inquiry and research design: choosing among five traditions. Thousand Oaks : SAGE, 1998.

FASSNACHT, G. Theory and practice of observing behavior. New York : Academic Press, 1982.

HEIDEGGER, Martin. Being and time. New York : Harper and Row Publisher, 1962.

HIRSCHMAN, Elizabeth C. e HOLBROOK, Morris B. Advances in consumer research. Provo. Utah : Association for Consumer Research, 1985.

HUSSERL, Edmond. The crisis of european sciences and transcendental phenomenology. Evanston : Northwestern University Press, 1970.
JACKSON, Michael. Things as they are: new directions in phenomenological anthropology. Bloomington : Indiana University Press, 1996.

MORSE, Janice M. Critical issues in qualitative research methods. London : SAGE, 1994.

MOUSTAKAS, C. Phenomenological research methods. Thousand Oaks : SAGE, 1994.

SHERRY, John F. Jr. Contemporary Marketing and consumer behavior: an anthropological sourcebook. Thousand Oaks : SAGE, 1995.

VAN MANEN, Max. Researching lived experience: human science for an action sensitive pedagogy. New York : State University of New York Press, 1990.

YIN, Robert K. Case study research: design and methods. $2^{\text {nd }}$ ed. Thousand Oaks, CA: SAGE, 1994.

ZALTMAN, Gerald, LEMASTERS, Karen e HEFFRING, Michael. Theory construction in Marketing, some thoughts on thinking. New York : John Wiley \& Sons, 1982. 
xos estudos que envolvem, como enfocou-se aqui, as situações interativas que têm lugar nos espaços de serviços.

Alguns autores acreditam que as abordagens tradicionais de mercadologia vêm-se mostrando insuficientes quando se trata de buscar compreender as complexas interações de serviços. Por outro lado, o aporte de perspectivas interpretativas, subjetivas e até mesmo críticas permitidas pelas possibilidades interdisciplinar e transdisciplinar da pesquisa em Marketing, entre as quais classifica-se a abordagem fenomenológica, parece anunciar novos e interessantes caminhos para os acadêmicos envolvidos no estudo do fenômeno de serviços.

Curiosamente, até o final dos anos 1990, tais perspectivas eram escassas na produção científica voltada para o estudo dos ambientes físicos de serviços; a partir da segunda metade da década passada, contudo, intensificando-se o aporte de construções teóricas do campo da Antropologia e da Semiologia para a Administração de Marketing, transformaram-se substancialmente as possibilidades de compreensão sócio-técnica dos cenários mercadológicos. Na prática, talvez essas al- ternativas - cuja utilização ainda encontra-se comprometida pelas deficiências de formação e aplicação das perspectivas subjetivistas em Administração - venham a se tornar mais presentes à medida que forem sendo oferecidas, nos cursos de mestrado e doutorado, disciplinas metodológicas obrigatórias ou eletivas que se esforcem para tratar da dimensão qualitativa da pesquisa acadêmica em Marketing como algo além da simples curiosidade científica.

$\mathrm{O}$ presente ensaio objetivou fornecer indicações de procedimentos metodológicos básicos para a pesquisa fenomenológica, uma opção para investigação dos espaços de serviços. Motivou-o a crença de que fenômenos interativos, como o marketing de serviços, não podem ser amplamente captados por métodos quantitativos. O artigo ofereceu indicações de problemas de pesquisa e sugestões para a coleta e o tratamento dos dados, alertando o leitor, no entanto, para as limitações e dificuldades do método fenomenológico. Espera-se que outros estudiosos sensibilizem-se para a pertinência do método e, adotando-o, registrem suas experiências de modo a enriquecer o parco acervo existente. $\bigcirc$

\section{REFERÊNCIAS BIBLIOGRÁFICAS \\ ALLAN, K. A formalization of postmodern theory. Sociological Perspectives, v. 43, n. 3, Fall 2000. \\ BERTERO, C. O., CALDAS, M. P., WOOD JR., T. Produção científica em administração de empresas: provocações, insinuações e contribuições para um debate local. Revista de Administração Contemporânea, v. 3, n. 1, 1999.}

ALVESSON, M., DEETZ, S. Teoria crítica abordagens pós-modernas para estudos organizacionais. In: CLEEG, HARDY, NORD (Orgs.). Handbook de estudos organizacionais. São Paulo : Atlas, 1999.

ARNOULD, E. J., PRICE, L. L. River magic: extraordinary experience and the extended service encounter. Journal of Consumer Research, v. 26, 1993.

AUBERT-GAMET, V., COVA, B. Servicescapes: from modern non-places to postmodern common places. Journal of Business Research, v. 44, Special Issue "Service Marketing and Management - European Contributions", 1999.

BAKER, J. Examining the informational value of store environments, servicescapes: the concept of place in contemporary markets. In: SHERRY JR., J. F. (Ed.).Servicescapes: the concept of place in contemporary markets. Chicago : NTC Business Books, 1998.
BITNER, M. J. Servicescapes: the impact of physical surroundings on customer and employees. Journal of Marketing, v. 56, n. 2, 1992

BOTELHO, D., MACERA, A. Análise metateórica de teses e dissertações da área de marketing apresentadas na EAESP-FGV (1974-1999). In ENCONTRO NACIONAL DA ASSOCIAÇÃO NACIONAL DOS PROGRAMAS DE PÓS-GRADUAÇÃO EM ADMINISTRAÇÃO, 25., 2001, Campinas. Anais.. Campinas: ANPAD, 2001

BROWN, S. Postmodern marketing? European Journal of Marketing, v. 27, n. 4, 1993.

BROWN, S. A postmodern world: introduction to the special issue. European Journal of Marketing, v. 31 n. $3 / 4,1997$

BROWN, S., FISK, R., BITNER, M. J. The development and emergence of services marketing thought. International Journal of Service Industry Management, v. 5, n. 1,1994
BROWNLIE, D. Beyond ethnography - towards writerly accounts of organizing in marketing. European Journal of Marketing, v. 31, n. 3/4, 1997.

BROWNLIE, D., SAREN, M. The four Ps of the marketing concept: prescritive, polemical, permanent and problematical. European Journal of Marketing, v. 26, n. 4, 1992.

BROWNLIE, D., SAREN, M., WENSLEY, R. WHITTINGTON, R. Marketing disequilibrium: on redress and restoration. In: BROWNLIE et. al. Rethinking marketing. London: Sage, 1999.

BUNGE, M. Philosophy of science: from problem to theory (Rev. Ed.), V. I. New Brunswick: Transaction, 1998.

BURRELL, G., MORGAN, G. Sociological paradigms and organisational analysis. England: Heinemann, 1979.

CARVAlhO, J. L. F. S., MOTTA, P. C. Iluminando cenários de serviços: um exame das funções da luz nas "horas da verdade". In: ENCONTRO NACIONAL DA ASSOCIAÇÃO NACIONAL DOS PROGRAMAS DE PÓS-GRADUAÇÃO EM ADMINISTRAÇÃO, 24., 2000, Florianópolis. Anais... Florianópolis : ANPAD, 2000. 
CARVALHO, J. L. F. S., VERGARA, S. C. Repensando os roteiros de marketing de serviços: análise crítica da teoria dos scripts cognitivos. In: ENCONTRO NACIONAL DA ASSOCIAÇÃO NACIONAL DOS PROGRAMAS DE PÓS-GRADUAÇÃO EM ADMINISTRAÇÃO, 24., 2000, Florianópolis. Anais... Florianópolis: ANPAD, 2000.

CASOTTI, L. O que é a pesquisa do consumidor? Reflexões geradas a partir de umproblema prático. In: ENCONTRO NACIONAL DA ASSOCIAÇÃO NACIONAL DOS PROGRAMAS DE PÓSGRADUAÇÃO EM ADMINISTRAÇÃO, 23., 1999, Foz do Iguaçu. Anais... Foz do Iguaçu : ANPAD, 1999.

CAVEDON, N. R. Recursos metodológicos e formas alternativas no desenvolvimento e na apresentação de pesquisas em administração. In: ENCONTRO NACIONAL DA ASSOCIAÇ̃̃O NACIONAL DOS PROGRAMAS DE PÓS-GRADUAÇÃO EM ADMINISTRAÇÃO, 25., 2001, Campinas. Anais.. Campinas: ANPAD, 2001

CHIA, R. From modern to postmodern organizational analysis. Organization Studies, v. 16 n. 4, 1995.

CHURCHILL, S. D., WERTZ, F. J. An introduction to phenomenological psychology for consumer research: historical, conceptual, and methodological foundations. In: HIRSCHMAN, E. C., HOLBROOK, M B. (Eds.) Advances in Consumer Research. Provo : Association for Consumer Research, 1985.

COVA, B. What postmodernism means to marketing managers. European Management Journal, v. 14, n. 5, 1996

COVA, B. Community and consumption: towards a definition of the "linking value" of products and services. European Management Journal, v. 31, n. 3/4, 1997

COVA, B., BADOT, O. Marketing theory and practice in a postmodern era. In: BAKER, M. J. Marketing theory and practice. $3^{\text {rd }}$ ed. Basingstoke : MacMillan, 1995.

CREIGHTON, M. The seed of creative lifestyle shopping: wrapping consumerism in japanese store layouts. In: SHERRY JR., J. F. (Ed.) Servicescapes: the concept of place in contemporary markets. Chicago : NTC Business Books, 1998.

CRESWELL, J. W. Qualitative inquiry and research design: choosing among five traditions. Thousand Oaks : Sage Publications, 1998.

DONOVAN, R. J., ROSSITER, J. R. Store atmosphere: an environmental psychology approach. Journal of Retailing, v. 58, n. 1, Spring 1982.
FARIAS, S. A., GÓIS, G. T., OLIVEIRA, L. A. C.. Por que isso ocorreu? Utilizando a teoria da atribuição para explicar as saídas no consumo de serviços. In ENCONTRO NACIONAL DA ASSOCIACCÃO DOS PROGRAMAS DE PÓS-GRADUAÇ̃̃O EM ADMINISTRAÇÃO, 24., 2000, Florianópolis. Anais... Florianópolis: ANPAD, 2000.

FOXALL, G. R. Science and interpretation in consume research: a radical behaviourist perspective. European Journal of Marketing, v. 29, n. 9, 1995.

GIL, A. C. Métodos e técnicas de pesquisa social. São Paulo: Atlas, 1987

GOTTDIENER, M. Semiotics and postmodernism. In: DICKENS, D. R., FONTANA, A. (Eds.) Postmodernism and social enquiry. New York: Guilford Press, 1994.

GOTTDIENER, M. Postmodern semiotics. Cambridge : Blackwell, 1995.

GOTTDIENER, M. The semiotics of consumer spaces: the growing importance of themed environments. In: SHERRY JR., J. F. (Ed.) Servicescapes: the concept of place in contemporary markets. Chicago: NTC Business Books, 1998.

GOULDING, C. Consumer research, interpretative paradigms and methodological ambiguities. European Journal of Marketing, v. 33, n. 9/10, Sept./Oct. 1999.

GRÖNROOS, C. Marketing : gerenciamento e serviços. Rio de Janeiro : Campus, 1995

GROVE, S. J., FISK, R. P., DORSCH, M. J. Assessing the theatrical components of the service encounter: a cluster analysis examination. The Service Industries Journal, v. 18, n. 3, 1998

HABERMAS, J. La technique et la science comme idéologie. Paris: Gallimard, 1963.

HEIDEGGER, M. Being and time. New York : Harper and Row Publisher, 1962.

HELGESON, J. G. Receiving and responding to a mail survey: a phenomenological examination. Journal of the Market Research Society, v. 36, n. 4, Oct. 1994.

HUSSERL, E. The crisis of european sciences and transcendental phenomenology. Evanston: Northwestern University Press, 1970.
ICHIKAWA, E. Y., SANTOS, L. W. Apresentando a Grounded Theory: uma nova proposta de abordagem qualitativa na pesquisa organizacional. In: ENCONTRO NACIONAL DA ASSOCIACC̃O NACIONAL DOS PROGRAMAS DE PÓS-GRADUAÇÃO EM ADMINISTRAÇÃO, 25., 2001, Campinas. Anais.. Campinas: ANPAD, 2001

JACKSON, M. Things as they are: new directions in phenomenological anthropology. Bloomington : Indiana University Press, 1996.

KERLINGER, F. N., LEE, H. B. Foundations of behavioral research, $4^{\text {th }}$ ed. Orlando: Harcourt College Publishers, 2000.

KILDUFF, M., MEHRA, A. Postmodernism and organisational research. Academy of Management Review, v. 22, n. 2, April 1997.

LEVY, M., WEITZ, B. A. Administração de varejo. São Paulo: Atlas, 2000

LIMA, J. B. Pesquisa qualitativa e qualidade na produção científica em administração de empresas. In: ENCONTRO NACIONAL DA ASSOCIAÇÃO NACIONAL DOS PROGRAMAS DE PÓS-GRADUAÇÃO EM ADMINISTRAÇÃO, 23., 1999, Foz do Iguaçu. Anais... Foz do Iguaçu : ANPAD, 1999

LOVELOCK, C., WRIGHT, L. Serviços: marketing e gestão. São Paulo: Saraiva, 2001.

MALHOTRA, N. K., PETERSON, M. Marketing research in the new millenium: emerging issues and trends. Marketing Intelligence \& Planning, v. 19, n. 4, 2001

MARTINS, G. A. Epistemologia da pesquisa em administração. In: ENCONTRO NACIONAL DA ASSOCIAÇÃO NACIONAL DOS PROGRAMAS DE PÓS-GRADUAÇÃO EM ADMINISTRAÇÃO, 20., 1996، Salvador. Anais... Salvador : ANPAD, 1996.

MASINI, E. F. S. Enfoque fenomenológico de pesquisa em educação. In: FAZENDA, I. (Ed.) Metodologia da pesquisa educacional. São Paulo: Cortez, 1989

MENDONÇA, J. R. C. Interacionismo simbólico: uma sugestão metodológica para a pesquisa em administração. In: ENCONTRO NACIONAL DA ASSOCIAÇÃO NACIONAL DOS PROGRAMAS DE PÓS-GRADUAÇÃO EM ADMINISTRAÇÃO, 25., 2001, Campinas. Anais... Campinas: ANPAD, 2001.

MILES, M. B., HUBERMAN, A. M. Qualitative data analysis: a sourcebook of new methods. $2^{\text {nd }}$ ed. Beverly Hills : Sage Publications, 1994. 
MILLIKEN, J. Qualitative research and marketing management. Journal of Management Decision, v. 39, n.1, 2001.

MILLS, C. W. A imaginação sociológica. $6^{\mathrm{a}}$ ed. Rio de Janeiro: Zahar, 1982.

MORENTE, M. G. Fundamentos de filosofia. 8. ed. São Paulo : Mestre Jou, 1980.

MORSE, J. M. Emerging from the data: the cognitive process of analysis in qualitative inquiry. In: MORSE, J. M. (Ed.) Critical issues in qualitative research methods. London : Sage Publications, 1994.

MOTTA, P. C. Serviços: pesquisando a satisfação do consumidor. Rio de Janeiro : Papel Virtual, 1999.

MOTTA, P. C. Servir com alma - um novo conceito em relacionamento com o cliente. São Paulo: Prentice Hall, 2000.

MOUSTAKAS, C. Phenomenological research methods. Thousand Oaks: Sage Publications, 1994.

NICOLINI, A. Qual será o futuro das fábricas de administradores? In: ENCONTRO NACIONAL DA ASSOCIAÇÃO NACIONAL DOS PROGRAMAS DE PÓS-GRADUAÇÃO EM ADMINISTRAÇÃO, 25., 2001, Campinas. Anais... Campinas: ANPAD, 2001.

NORMANN, R. Administração de serviços: estratégia e liderança na empresa de serviços. São Paulo : Atlas, 1993.

OAKES, S. The influence of the musicscape within service environments. Journal of Services Marketing, v. 14, n. 7, 2000.

PATTON, M. Q. Qualitative evaluation and research methods. $2^{\text {nd }}$ ed. London: Sage Publications, 1990.

PHILIPS, N. Telling organizational tales: on the role of narrative fiction in the study of organizations. Organization Studies, v. 16, n. 4, 1995.

PIRSIG, R. M. Zen e a arte da manutenção de motocicletas. $7^{a}$ ed. São Paulo: Paz e Terra, 1987.

PRUS, R. Just browsing, thanks! Focused and diffuse shopping practices. Winter's Educational American Marketing Association Conference, Orlando, Feb. 1991.
RAY, M. A. The richness of phenomenology: philosophic, theoretic and methodologic concerns. In: MORSE, J. M. (Ed.) Critical issues in qualitative research methods. London : Sage Publications, 1994.

REMENYI, D., WILLIAMS, B., MONEY, A., SWARTZ, E. Doing research in business and management: an introduction to process and method. London : Sage Publications, 1998.

RÉVILLION, A. S. P. A utilização de pesquisas exploratórias na área de marketing. In: ENCONTRO NACIONAL DA ASSOCIAÇÃO NACIONAL DOS PROGRAMAS DE PÓS-GRADUAÇÃO EM ADMINISTRAÇÃO, 25., 2001, Campinas. Anais.. Campinas: ANPAD, 2001.

REYNOLDS, P. D. A primer in theory construction. New York : Bobbs-Merril, 1971.

ROBSON, I., ROWE, J. Marketing - the whore of Babylon? European Journal of Marketing, v. 31, n. 9/ 10, 1997.

ROSSI, C. A. V., HOR-MEYLL, L. F. Explorando novas trilhas na pesquisa do consumidor. In: ENCONTRO NACIONAL DA ASSOCIACÃO NACIONAL DOS PROGRAMAS DE PÓS-GRADUAÇÃO EM ADMINISTRAÇÃO, 25., 2001, Campinas. Anais.. Campinas: ANPAD, 2001

SANDIKCI, O., HOLT, D. B. Malling society: mall consumption practices and the future of public space. In: SHERRY JR., J. F. (Ed.) Servicescapes: the concept of place in contemporary markets. Chicago : NTC Business Books, 1998.

SANTOS, U. W. B. Métodos qualitativos para pesquisa em administração: caracterização e relacionamento aos paradigmas para pesquisa. Dissertação (Mestrado) - Departamento de Administração de Empresas, PUCRio de Janeiro, 1994.

SCHOUTEN, J. W., MCALEXANDER , J. H. Subcultures of consumption: an ethnography of the new bikers. Journal of Consumer Research, v. 22, June 1995

SERVA, M., JAIME JR., P. Itinerários teóricos e práticas profissionais numa fronteira interdisciplinar: contribuições para o ensino em administração. In: ENCONTRO NACIONAL DA ASSOCIAÇÃO NACIONAL DOS PROGRAMAS DE PÓS-GRADUAÇÃO EM ADMINISTRAÇÃO, 25., 2001, Campinas.Anais... Campinas : ANPAD, 2001.
SHERRY JR., J. F. Understanding markets as places: an introduction to servicescapes In: SHERRY JR., J. F. (Ed.) Servicescapes: the concept of place in contemporary markets. Chicago : NTC Business Books, 1998.

SHERRY JR., J. F. Anthropology of marketing and consumption: retrospect and prospect. In: SHERRY JR., J. F. (Ed.) Contemporary marketing and consumer behavior: an anthropological sourcebook. Thousand Oaks: Sage Publications, 1995.

SOKAL, A., BRICMONT, J. Imposturas intelectuais: o abuso da ciência pelos filósofos pós-modernos. Rio de Janeiro: Record, 1999.

SOLOMON, M. R. Dressing for the part: the role of costume in the staging of the servicescape. In: SHERRY JR., J. F. (Ed.) Servicescapes: the concept of place in contemporary markets. Chicago : NTC Business Books, 1998.

SZMIGIN, I., FOXALL, G. R. Interpretive consumer research: how far have we come? Qualitative Market Research International, v. 3, n. 4, 2000.

THOMPSON, C. L., LOCANDER, W. B., POLLIO, H. R. Putting consumer experience back into consumer research: the philosophy and method of existencialphenomenology. Journal of Consumer Research, $v$. 16, n. 12, Sept. 1989.

THORNE, S. Doing qualitative research. Qualitative Health Research, v. 11, n. 1, Jan. 2001.

VAN MANEN, M. Researching lived experience: human science for an action sensitive pedagogy. New York : State University of New York Press, 1990.

VERGARA, S. C. Teoria prática educacional - da técnica à ética. Revista Fórum Educacional, v. 13, n. 3, jun./ago. de 1989.

VERGARA, S. C. Projetos e relatórios de pesquisa em administração. 3. ed. São Paulo : Atlas, 2000.

WEIL, P., D’AMBROSIO, U., CREMA, R. Rumo à nova transdisciplinaridade. São Paulo: Summus, 1993.

ZALTMAN, G. Consumer researchers: take a hike! Journal of Consumer Research, v. 26, n. 4, março de 2000 .

ZEITHAML, V. Consumer perceptions of Price, quality and value: a means-end model and synthesis of evidence. Journal of Marketing, v. 52, July 1988.

ZEITHAML, V. A., BITNER, M. J. Services marketing New York: McGraw-Hill, 1996. 